\title{
Una nueva especie de Trichomyia Haliday (Diptera, Psychodidae) de Los Montes de María, Colombia
}

\author{
Alveiro Pérez-Doria ${ }^{1}$, Ela Hernández ${ }^{1} \&$ Eduar Elías Bejarano $^{1}$
}

1Grupo de Investigaciones Biomédicas, Universidad de Sucre, Cra. 14 No. 16-B-32, A.A. 406, Sincelejo, Colombia. eduarelias@yahoo.com

\begin{abstract}
A new species of Trichomyia Haliday (Diptera, Psychodidae) from Los Montes de María, Colombia. Trichomyia colosensis n. sp. is described and illustrated from male specimens collected with a modified CDC light trap in the Reserva Forestal Protectora Serranía de Coraza y Montes de María, on the Caribbean Coast of Colombia. To date, two species of Trichomyia have been reported from the Colombian Caribbean, T. brevitarsa (Rapp, 1945) and T. colosensis n. sp.

KEY WORDS. Colombian Caribbean; psychodid fly; Trichomyiinae; taxonomy.

RESUMEN. Una nueva especie de Trichomyia Haliday (Diptera, Psychodidae) de Los Montes de María, Colombia. Trichomyia colosensis n. sp. es descrita e ilustrada a partir de ejemplares machos recolectados con una trampa modificada CDC en la Reserva Forestal Protectora Serranía de Coraza y Montes de María, sobre la Costa Caribe Colombiana. Hasta la fecha están registradas dos especies de Trichomyia en el Caribe colombiano, T. brevitarsa (Rapp, 1945) y T. colosensis $\mathbf{n}$. sp.
\end{abstract}

PALABRAS CLAVE. Caribe Colombiano; psicódido; Trichomyiinae; taxonomía.

Los psicódidos son insectos cosmopolitas agrupados taxonómicamente en cinco subfamilias, Phlebotominae Rondani, 1840, Psychodinae Rondani, 1856, Bruchomyiinae Alexander, 1920, Trichomyiinae Tonnoir, 1922, y Sycoracinae Jung, 1954, la penúltima de las cuales está compuesta por el género Trichomyia Haliday, 1839 (Bejarano 2006a). Las especies de Trichomyia se diferencian de los otros psicódidos por la combinación de los siguientes caracteres, la presencia de cuatro venas radiales, una sola vena longitudinal entre las bifurcaciones media y radial, y la extensión de la vena $\mathrm{CuA}$ hasta o más allá de la bifurcación media del ala (Quate 1996).

Dentro de la subfamilia Trichomyiinae se pueden distinguir dos grupos morfológicos, con base en el número de segmentos que conforman los pálpos maxilares, aunque las relaciones evolutivas entre ellos no han sido completamente esclarecidas (Duckhouse 1978; Bravo 2000). El primer grupo está compuesto por las especies que poseen tres segmentos palpales, las cuales se encuentran distribuidas por todo el mundo, mientras que el segundo grupo está integrado por las especies que presentan los palpos divididos en cuatro segmentos. Entre las últimas, que también tienen una distribución mundial, es posible distinguir un pequeño subgrupo constituido por especies con el primer y segundo palpómero parcialmente fusionados, las cuales se encuentran restringidas al Neotrópico (Bravo 2000).

El género Trichomyia fue registrado por primera vez en Colombia por Bejarano (2006a), con base en ejemplares de T. brevitarsa (Rapp, 1945) encontrados en territorios insulares y continentales del norte del país. Más tarde, se informó el hallazgo de hembras del mismo género en la región Amazónica (Bejarano et al. 2009). El propósito de este trabajo es describir una nueva especie de Trichomyia de la Costa Caribe Colombiana.

\section{MATERIAL Y MÉTODOS}

Los psicódidos se recolectaron con una trampa modificada de luz CDC, en la Estación Experimental de Fauna Silvestre de la Reserva Forestal Protectora Serranía de Coraza y Montes de María, municipio de Colosó $\left(09^{\circ} 31^{\prime} 48.0^{\prime \prime} \mathrm{N}, 75^{\circ} 21^{\prime} 4.3^{\prime \prime} \mathrm{W}\right)$, departamento de Sucre, Colombia. Las modificaciones realizadas a la trampa consistieron en adecuarla a la operación con corriente eléctrica de $110 / 120 \mathrm{~V}$, y adaptarle una bombilla roja de $7.5 \mathrm{~W}, 120 \mathrm{~V}$, y un extractor tipo Sun Sp 200A, 115V, Ac- $50 / 60 \mathrm{~Hz}$ (Pérez-Doria et al. 2008). La trampa se instaló a una altura de $1.5 \mathrm{~m}$ y se activó entre las 18:00 y 06:00 horas. El área donde se desarrollaron los muestreos corresponde a un relicto de transición de bosque seco premontano a bosque seco tropical, con una altura de $300 \mathrm{msnm}$, una temperatura media por año de $27^{\circ} \mathrm{C}$ y una precipitación promedio de 1.200 $\mathrm{mm}$ anuales. Este sistema montañoso es considerado como la continuación de la Cordillera Occidental de Los Andes en el norte de Colombia.

Cada psicódido se trató durante 24 horas, con una solución de ácido láctico y fenol, en proporción 1:1, para facilitar la observación de las estructuras de interés taxonómico. Los ejemplares fueron luego cuidadosamente diseccionados bajo un estereomicroscopio Carl Zeiss Stemi DV4, donde se separó la cabeza, el tórax, un ala y el abdomen. Posteriormente, los especímenes se montaron, de forma temporal, sobre láminas portaobjeto con bordes recubiertos por parafina, usando la misma solución de lactofenol, y se fotografiaron con una cámara digital Canon A640 acoplada a un microscopio triocular Carl Zeiss Primo Star. Una vez obtenidas las fotografías e ilustraciones, se realizaron preparaciones permanentes de los ejemplares con bálsamo de Canadá. Cada variable morfométrica fue estimada con un micrómetro de 
ocular, calibrado con un micrómetro de objeto Carl Zeiss 5 $+100 / 100 \mathrm{~mm}$. Las medidas del ala se establecieron con el programa Image Pro-Plus ${ }^{\circledR}$, el cual también fue calibrado con el micrómetro de objeto descrito. La nomenclatura usada corresponde, en parte, a la propuesta por McAlpine (1981). El material tipo reposa en la Colección de Artrópodos de Importancia Médica de la Universidad de Sucre - CAIMUS, en Sincelejo, Colombia.

\section{RESULTADOS}

\section{Trichomyia colosensis $\mathbf{n}$. sp.}

(Figs. 1-8)

Descripción (todas las medidas están expresadas en micras). Psicódido con una longitud de 1086 desde el borde anterior del prescuto hasta los genitales. Cabeza, tórax y abdomen fuertemente pigmentados de color marrón oscuro.

Cabeza. Vértice recubierto por cicatrices de setas entremezcladas con pequeñas pilosidades, con el frente revestido sólo por estas últimas. Hilera de cicatrices grandes de setas bordeando el margen interno del ojo. Cabeza más ancha que larga (Figs. 1,2), con una altura desde el vértice hasta el borde distal del clípeo de 285 y una anchura de 420 . Clípeo más ancho que largo, sin extenderse más allá del nivel de los ojos, recubierto por pilosidades y con 25 cicatrices de setas. Anchura del clípeo 98, con una altura de 55. Labroepifaringe con 10 papilas en su mitad proximal y cuatro setas sensoriales en la distal, dos de las últimas situadas en la punta. Labio con seis setas espiniformes cerca del ápice. Longitud del labro-epifaringe 70, medida de los labios 87. Faringe sin espinas (Fig. 2). Ojos compuestos, sin puente ocular, con una altura de 260. Distancia interocular 140, equivalente al diámetro de 5.9 facetas. Escapo y pedicelo subesféricos (Fig. 1), con presencia de dos a tres hileras irregulares de cicatrices de setas. Anchura del escapo 60, con 35 de longitud en su parte media. Base del escapo con dos setas sensoriales y una prolongada dilatación lateral, que le permite articularse con la cabeza, y ápice con una dilatación lateral pequeña, acoplada al pedicelo. Pedicelo con una longitud y anchura de 53. Flagelómeros cortos, piriformes (Fig. 3) y recubiertos en su mitad proximal por pelos largos. Longitud de los flagelómeros: I 80, II 88, III 93, IV 100 y V 100. El ancho de los flagelómeros disminuye desde el flagelómero I que mide 48 hasta el flagelómero II que mide 36, en los artejos III, IV y V la anchura se mantiene constante. Segmentos proximales de la antena articulados entre si de manera casi céntrica, en contraste con los artejos distales que se unen excéntricamente. Antenas incompletas apicalmente. Ascoides pareados, gruesos y sutilmente estriados, insertados al mismo nivel, extendiéndose desde la base del flagelómero hasta la mitad distal del siguiente segmento (Fig. 3). Palpos maxilares con tres segmentos, recubiertos por setas foliáceas y con setas espiniformes en el segundo y tercer palpómero. Longitud de los palpómeros: primero 63, segundo 46, tercero 41, en proporción 1.0-0.73-0.65. Formula palpal 3.2.1. Palpómero primero con $\approx 15$ sensilas de ápice no dilatado, agrupadas en una base circular sobre el lado interno del segmento.
Tórax. Prescuto, escuto, escutelo, coxas y parte de la pleura, fuertemente pigmentados de color marrón oscuro. Alas con el ápice redondeado (Fig. 7). Longitud del ala desde el inicio de la vena costa 1404, anchura máxima 541. Proporción entre la longitud del ala y su anchura máxima 2.6. Longitud de las venas principales del ala: $R_{1} 539, R_{2}+R_{3} 335, R_{4} 481$, $\mathrm{R}_{5}$ 906, $\mathrm{M}_{1} 649, \mathrm{M}_{2}$ 492, $\mathrm{M}_{3} 663, \mathrm{M}_{4}$ 527, CuA 470. Vena humeral ausente. Vena Sc discontinua. Bifurcaciones radial y media separadas por 180 . Venas $R_{1}, R_{2}+R_{3}, M_{2}, M_{3}, M_{4} y$ $\mathrm{CuA}$ tocando débilmente el margen del ala. Vena transversal $\mathrm{r}-\mathrm{m}$ ausente. Vena CuA larga, extendiéndose hasta el nivel de la bifurcación media. Vena transversal $\mathrm{m}$-cu ausente. Venas $\mathrm{R}_{1} \mathrm{y}_{4}$ engrosadas. Halterios de color claro, cubiertos por numerosas cicatrices de setas. Longitud del halterio 200, anchura máxima 90. Proporción entre la longitud del halterio y su anchura máxima 2.2.

Abdomen. Abdomen de color marrón oscuro, con una longitud de 649, incluidos los órganos genitales, y revestido por cicatrices de setas foliáceas. Tergito $10 \mathrm{y}$ esternito 10 recubiertos sólo por finas pilosidades, cercos con setas espiniformes. Tergito 9 grande y subcuadrado, tergito 10 pequeño y triangular, esternito 10 grande, triangular y con la punta achatada (Fig. 8). Genitales relativamente complejos (Figs. 5,6). Cerco, en visión dorso-ventral, con dos prominentes prolongaciones fuertemente esclerotizadas, apical y subapical, dirigidas hacia adentro (Fig. 8). Lateralmente, la prolongación subapical se puede observar como un pequeño apéndice en el margen anterior del cerco, mientras la prolongación apical sobresale, ampliamente, en la punta de éste (Fig. 6). Gonocoxito con una prolongación distal ventral que, en visión dorso-ventral, corre paralela al aedeago, sin llegar al ápice de éste, y una prolongación distal dorsal recubierta por setas espiniformes, que le dan a la estructura un aspecto espinoso (Fig. 5). Prolongación distal dorsal con dos apéndices laterales digitiformes de desigual longitud, en cada uno de los cuales se implanta una larga espina. En visión dorso-ventral, la espina del apéndice digitiforme mayor, es recta, pero se dobla abruptamente antes de la punta, a diferencia de la espina del apéndice digitiforme menor que es curva (Fig. 5). Cerca de la base de los apéndices digitiformes, se insertan otras espinas de menor tamaño. Ápice de la prolongación distal dorsal recubierto por setas gruesas, de color negro y comprimidas, que le otorgan un aspecto dentado (Fig. 5). Gonoestilo de tamaño pequeño, con bordes esclerotizados, puntiagudo en visión lateral (Fig. 6), y con forma subtriangular, en visión dorso-ventral (Fig. 4). Parámero fuertemente esclerotizado (Fig. 6), con dos pequeñas prolongaciones transversales, una cerca de su base y otra en la mitad, y finalizando en una punta gruesa y roma dirigida lateralmente (Fig. 5). Aedeago con bordes esclerotizados terminando en una sola punta (Figs. 5,6). Bomba genital fuertemente esclerotizada, con aspecto cilíndrico, en visión dorso-ventral (Fig. 5). Apodema eyaculador esclerotizado, alargado en visión dorso-ventral y redondeado en visión lateral (Fig. 6).

Material Tipo. Holotipo: 1 macho, COLOMBIA, Sucre, Colosó (Estación Experimental de Fauna Silvestre, Reserva Forestal Protectora 


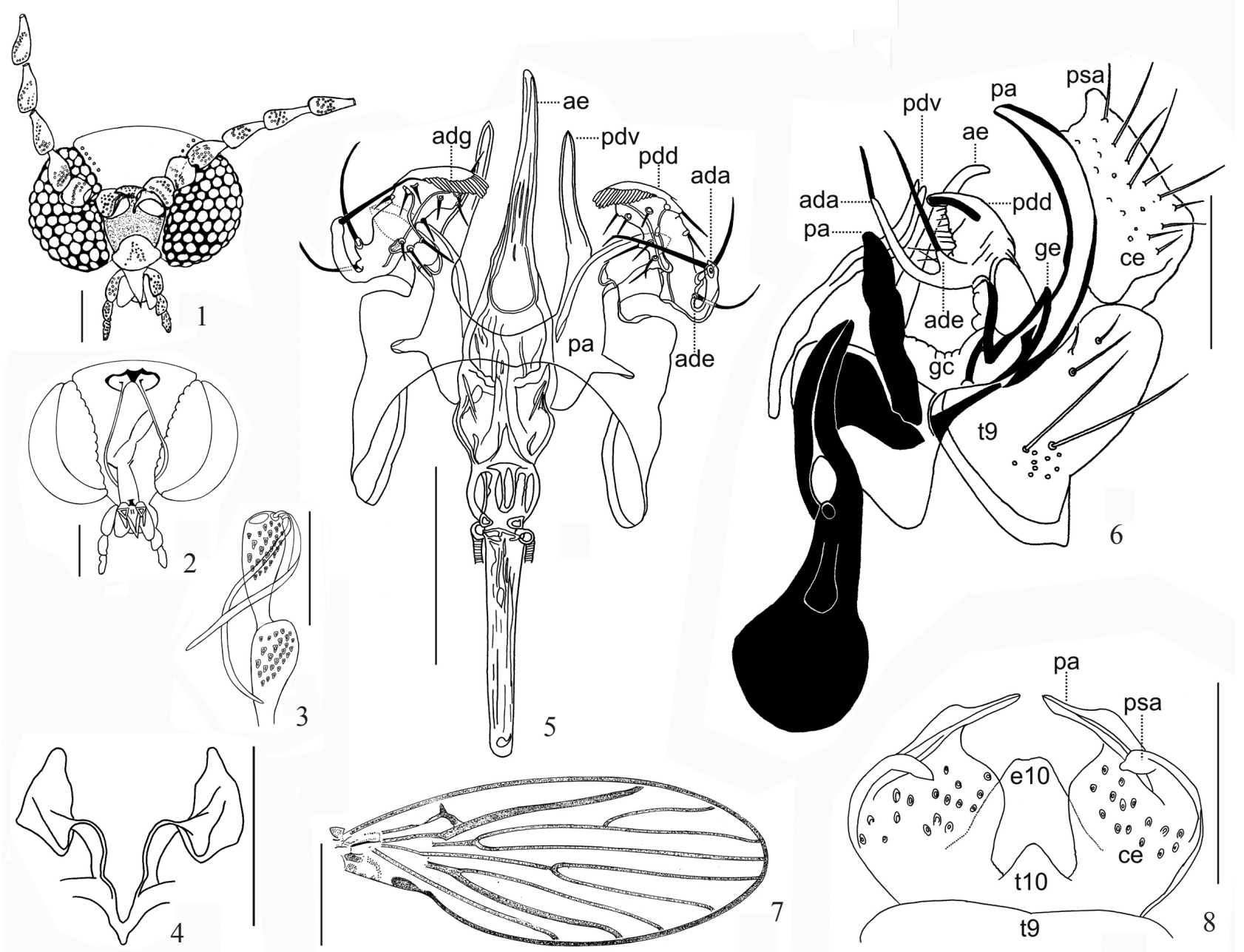

Figs. 1-8. Trichomyia colosensis $\mathbf{n}$. sp., paratipo macho: 1 , cabeza en visión frontal, escala $=100 \mu \mathrm{m}$; 2 , cabeza en visión dorsal, escala $=100 \mu \mathrm{m}$; 3 , flagelómeros 3 y 4, escala $=100 \mu \mathrm{m}$; holotipo macho: 4, gonoestilos en visión dorso-ventral, escala $=100 \mu \mathrm{m} ; 5$, genitalia en visión dorso-ventral, escala $=$ $100 \mu \mathrm{m} ; 6$, genitalia en visión lateral, escala $=100 \mu \mathrm{m} ; 7$, ala, escala $=500 \mu \mathrm{m} ; 8$, cercos, tergitos y esternito, escala $=100 \mu \mathrm{m}$. (ae, aedeago; ge, gonoestilo; gc, gonocoxita; pdv, prolongación distal ventral de la gonocoxita; pdd, prolongación distal dorsal de la gonocoxita; adg, setas comprimidas en forma dentada, sobre el ápice de la prolongación distal dorsal; ada, apéndice digitiforme mayor; ade, apéndice digitiforme menor; pa, parámero; ce, cerco; pa, prolongación apical del cerco; psa, prolongación subapical del cerco; t9, tergito 9; t10, tergito 10; e10, esternito 10).

Serranía de Coraza y Montes de María) 30.IV.2007, trampa de luz tipo CDC modificada, E. Hernández col. (CAIMUS). Paratipos: 1 macho, mismos datos que el holotipo, excepto 19-IV-2007; 1 macho, mismos datos que el holotipo, excepto 01-12-2008.

Etimología. El nombre de la especie proviene del topónimo Colosó, que se deriva del Cacique Onné Colosó, perteneciente a los Zenúes, el cual gobernó durante la época precolombina, la zona donde se recolectó el material entomológico tipo.

\section{DISCUSIÓN TAXONÓMICA}

La monogenérica subfamilia Trichomyiinae está representada en el Nuevo Mundo por 76 especies, incluidas 66 del Neotrópico (Duckhouse 1972, 1973; Wagner 1993, 1999; Wagner \& Masteller 1996; Quate 1996, 1999; Bravo 1999, 2000, 2001a, b, c, 2002; Alexander et al. 2001; IbáñezBernal 2004), 4 del Neártico (Wagner 1980) y 6 fósiles del ámbar mexicano (Quate 1961, 1963). Entre las especies neotropicales de Trichomyia, 29 se caracterizan por tener los palpos divididos en tres segmentos, rasgo compartido por T. colosensis n. sp. Sin embargo, el nuevo taxón puede ser diferenciado de sus congéneres por exhibir dos prolongaciones gruesas y esclerotizadas en la punta del cerco. $T$. colosensis n. sp. se distingue, igualmente, por el aspecto espinoso de la prolongación distal dorsal del gonocoxito, en el que se observan dos desiguales apéndices laterales digitiformes que terminan en espinas largas y delgadas.

Adicionalmente, es destacable la presencia de un grupo de setas comprimidas y negras, que le otorgan una apariencia dentada al ápice de la prolongación distal dorsal del gonocoxito. Es importante notar que este último carácter también está presente en las especies americanas $T$. bou Bravo, 1999, T. cauga Bravo, 1999 y T. mishi Bravo, 1999 (Bravo 1999), las cuales se diferencian de T. colosensis n. sp. por tener dos lóbulos laterales en el tergito siete, de los cuales se desprenden entre tres y cinco setas largas y esclerotizadas, que pueden unirse formando un haz grueso. Más aún, existen registros de la presencia de setas comprimidas, en forma semejante a lo observado en $T$. colosensis $\mathbf{n}$. sp., sobre el 
gonocoxito de taxones del Viejo Mundo (Duckhouse 1978), pero aún falta establecer el significado filogenético de este rasgo.

Hasta la fecha se han identificado seis subgéneros de Trichomyia, incluidos Apotrichomyia Duckhouse, 1978, Dactylotrichomyia Duckhouse, 1978, Dicrotrichomyia Duckhouse, 1978, Gondwanotrichomyia Duckhouse, 1980, Septemtrichomyia Bravo, 1999, y Opistotrichomyia Bravo, 2001, los dos últimos con representación en el Nuevo Mundo. Sin embargo, las características morfológicas de T. colosensis n. sp., impiden su inclusión en cualquiera de los subgéneros antes mencionados. Por último, el nuevo taxón puede ser distinguido fácilmente de $T$. brevitarsa, la otra especie de Trichomyia registrada hasta la fecha en la costa Caribe de Colombia (Bejarano 2006a), por tener los palpos divididos en tres segmentos, en adición a otros caracteres de los genitales. El registro de sólo dos especies de Trichomyia en el Caribe colombiano, pone en evidencia la necesidad de desarrollar más investigaciones para ampliar el conocimiento de la fauna de psicódidos del país, que con excepción de las especies hematófagas (Bejarano 2006b), ha sido poco estudiada hasta el momento.

\section{REFERENCIAS}

Alexander, B.; J. M. Freitas \& L. W. Quate. 2001. Some Psychodidae (Diptera) from Atlantic forest in South-Eastern Brazil, with descriptions of Trichomyia dolichopogon sp. nov. and Trichomyia riodocensis sp. nov. Brazilian Journal of Biology 61: 467-474.

Bejarano, E. E. 2006a. Subfamilia Trichomyiinae (Psychodidae) en el territorio continental e insular colombiano. Acta Biológica Colombiana 11: $37-41$.

Bejarano, E. E. 2006b. Lista actualizada de los psicódidos (Diptera: Psychodidae) de Colombia. Folia Entomológica Mexicana 45: 47-56.

Bejarano, E. E.; D. Sierra \& I. D. Vélez. 2009. Género Trichomyia Haliday, 1839 (Diptera: Psychodidae) asociado con Ficus Linn. (Moraceae) en la Amazonia Colombiana. Acta Amazônica 39: 473-475.

Bravo, F. 1999. Septemtrichomyia, subgênero novo de Trichomyiinae Neotropical (Diptera, Psychodidae). Revista Brasileira de Entomologia 43: $1-7$.

Bravo, F. 2000. Descrição de uma espécie de Trichomyia (Diptera, Psychodidae) do sudeste brasileiro, com comentários sobre a genealogia do gênero. Acta Biológica Leopoldensia 22: 185-192.

Bravo, F. 2001a. Opisthotrichomyia, subgênero novo de Trichomyiinae (Diptera, Psychodidae) e descrição de três novas espécies do Brasil. Sitientibus, Série Ciências Biológicas 1: 50-55.
Bravo, F. 2001b. Sete novas espécies de Trichomyia (Diptera, Psychodidae) da Mata Atlântica do Nordeste do Brasil. Sitientibus, Série Ciências Biológicas 1: 121-130.

Bravo, F. 2001c. Trichomyia quatei (Diptera, Psychodidae), uma nova espécie do nordeste brasileiro. Acta Biológica Leopoldensia 23: 31-37.

Bravo, F. 2002. Novas espécies de Trichomyia (Diptera, Psychodidae) da Mata Attântica da Bahia, nordeste do Brasil. Iheringia, Série Zoologia 92: $57-67$.

Duckhouse, D. A. 1972. Psychodidae (Diptera, Nematocera) of South Chile, subfamilies Sycoracinae and Trichomyiinae. Transactions of the Royal Entomological Society of London 124: 231-268.

Duckhouse, D. A. 1973. Family Psychodidae. Subfamilies Bruchomyiinae, Trichomyiinae, Sycoracinae and Psychodinae. In: P. E. Vanzolini \& N. Papavero (eds.). A catalogue of the Diptera of the Americas South of the United States. São Paulo, Museu de Zoologia, Universidade de São Paulo, $29 \mathrm{p}$.

Duckhouse, D. A. 1978. Taxonomy, phylogeny and distribution of the genus Trichomyia (Diptera, Psychodidae) in Australia and New Guinea. Systematic Entomology 3: 197-243.

Ibáñez-Bernal, S. 2004. Notes on the known species of Trichomyia Haliday of Mexico, with the establishment of a synonymy and the description of a new species (Diptera: Psychodidae). Zootaxa 523: 1-14.

McAlpine, J. F. 1981. Morphology and terminology - Adults, p. 9-63. In: J. F. McAlpine; B. V. Peterson; G. E. Shewell; H. J. Teskey; J. R. Vockeroth \& D. M. Wood (eds.). Manual of Nearctic Diptera. Ottawa, Research Branch, Agriculture Canada, Monograph 27, volume 1, vi+674 p.

Pérez-Doria, A.; E. Hernández-Oviedo \& E. E. Bejarano. 2008. Lutzomyia (Diptera: Psychodidae) de la Reserva Forestal Serranía de Coraza y Montes de María, Colombia. Revista Colombiana de Entomología 34: 98-101.

Quate, L. W. 1961. Fossil Psychodidae (Diptera: Insecta) in Mexican amber; part I. Journal of Paleontology 35: 949-951.

Quate, L. W. 1963. Fossil Psychodidae (Diptera: Insecta) in Mexican amber, part 2 (Diptera: Insecta). Journal of Paleontology 37: 110-118.

Quate, L. W. 1996. Preliminary taxonomy of Costa Rican Psychodidae (Diptera), exclusive of Phlebotominae. Revista de Biología Tropical 44: $1-81$.

Quate, L. W. 1999. Taxonomy of Neotropical Psychodidae (Diptera) 3. Psychodines of Barro Colorado Island and San Blas, Panama, p. 405441. In: J. F. Burger (ed.). Contributions to the knowledge of Diptera. Memoirs on entomology, International, Volume 14. Gainesville, Associated Publishers, $648 \mathrm{p}$.

Wagner, R. \& E. C. Masteller. 1996. New moth flies (Diptera:Psychodidae) and a key to species from Puerto Rico. Proceedings of the Entomological Society of Washington 98: 450-464.

Wagner, R. 1980. The Neartic Trichomyiinae (Diptera, Psychodidae). PanPacific Entomologist 56: 273-276.

Wagner, R. 1993. On a collection of Psychodidae (Diptera) by Dr. L. Botosaneanu from some Caribbean Islands. Aquatic Insects 15: 109127.

Wagner, R. 1999. Psychodidae from the Dominican Republic: records and descriptions of new species (Insecta: Diptera). Journal of the Kansas Entomological Society 72: 233-245. 